PESQUIMAT, Revista de la F.C.M. de la

Universidad Nacional Mayor de San Marcos

Vol. XV N², pp. 7-14, Lima - Perú, Marzo 2013

\title{
FUNCIONES GENERALIZADAS DE FRONTERA
}

\author{
Claudio Fernando Balcazar Huapaya ${ }^{1}$ \& Maruja Yolanda Gavilán Gonzales ${ }^{2}$
}

Resumen: En este trabajo probaremos, que en general, la restricción de una función del espacio de Sobolev $H^{1}(\Omega)$ a la frontera del dominio $\Omega$ pertenece a $L^{2}(\partial \Omega)$; para el caso de frontera con la condición de regularidad de ser continua y Lipschitz.

Palabras clave: Funciones generalizadas de frontera, regularidad, condición de Lipschitz.

\section{GENERALIZED FUNCTIONS OF BOUNDARY}

\begin{abstract}
In this paper we prove that in general, the restriction of a function space of Sobolev $H^{1}(\Omega)$ the boundary of the domain $\Omega$ belongs to $L^{2}(\partial \Omega)$; for the case of boundary regularly provided to be continuous and Lipschitz.
\end{abstract}

Key words: Generalized functions of boundary, regularity,condition of Lipschitz.

\section{Introducción}

Las condiciones de frontera de una ecuación diferencial parcial, en general, no se puede interpretar en sentido puntual y si consideramos un espacio de Sobolev $H^{1}(\Omega)$, con $\Omega \subset \mathbb{R}^{n}, \Omega$ abierto este puede contener funciones no acotadas por ello buscamos una interpretación de la restricción de las funciones en $H^{1}(\Omega)$ a la frontera de $\Omega$.

La frontera de $\partial \Omega$ de un dominio $n$-dimensional $\Omega$ puede ser interpretado como un objeto $n-1$ dimensional o variedad. Cuando $n=1, \partial \Omega$ puede consistir de puntos distintos en una variedad del caso cero-dimensional; y las desigualdades de Sobolev dan condiciones bajo el cual los valores puntuales son bien definidos para funciones en un espacio de Sobolev y de este modo para valores de frontera en el caso de dimensión uno.

\section{Preliminares}

Comenzamos con el siguiente ejemplo que después se generalizará. Sea $\Omega$ el disco unitario en $\mathbb{R}^{2}$ :

$$
\Omega=\left\{(x, y): x^{2}+y^{2}<1\right\}=\{(r, \theta): 0 \leq r<1,0 \leq \theta<2 \pi\} .
$$

Sea $u \in \mathcal{C}^{1}(\bar{\Omega})$, y se considera su restricción a $\partial \Omega$ como sigue

$$
\begin{aligned}
u(1, \theta)^{2} & =\int_{0}^{1} \frac{\partial}{\partial r}\left(r^{2} u(r, \theta)^{2}\right) d r \\
& =\int_{0}^{1} 2\left(r^{2} u u_{r}+r u^{2}\right)(r, \theta) d r
\end{aligned}
$$

Por otro lado tenemos que $u \nabla u=u\left(\frac{\partial u}{\partial x}, \frac{\partial u}{\partial y}\right)$ entonces

$$
u \nabla u \cdot \frac{(x, y)}{r}=\frac{1}{r} u\left(x \frac{\partial u}{\partial x}+y \frac{\partial u}{\partial y}\right)
$$

${ }^{1}$ UNMSM, Facultad de Ciencias Matemáticas, e-mail: cbalcazarh@unmsm.edu.pe

${ }^{2}$ UNMSM, Facultad de Ciencias Matemáticas, e-mail: mgavilan@unmsm.edu.pe 


$$
\begin{aligned}
\frac{\partial u}{\partial x} & =\frac{\partial u}{\partial r} \frac{\partial r}{\partial x}=u_{r} \frac{\partial r}{\partial x}=u_{r} \frac{x}{r}, \\
\text { y } \frac{\partial u}{\partial y} & =\frac{\partial u}{\partial r} \frac{\partial r}{\partial y}=u_{r} \frac{\partial r}{\partial y}=u_{r} \frac{y}{r},
\end{aligned}
$$

sustituyendo (2.3) y (2.4) en (2.2)

$$
\begin{aligned}
\nabla u \cdot \frac{(x, y)}{r} & =\frac{1}{r} u\left(x u_{r} \frac{x}{r}+y u_{r} \frac{y}{r}\right) \\
& =\frac{1}{r} u\left(\frac{u_{r}}{r} x^{2}+\frac{u_{r}}{r} y^{2}\right) \\
& =\frac{1}{r^{2}} u u_{r}\left(x^{2}+y^{2}\right) \\
& =u u_{r} .
\end{aligned}
$$

Luego

$$
r^{2} u \nabla u \cdot \frac{(x, y)}{r}=r^{2} u u_{r},
$$

sustituyendo (2.5) en (2.1)

$$
\begin{aligned}
\left|u(1, \theta)^{2}\right| & =\left|\int_{0}^{1} 2\left(r^{2} u \nabla u \cdot \frac{(x, y)}{r}+r u^{2}\right)(r, \theta) d r\right| \\
& \leq \int_{0}^{1} 2\left(\left|r^{2} u \nabla u \cdot \frac{(x, y)}{r}\right|+|r|\left|u^{2}\right|\right)(r, \theta) d r \\
& =\int_{0}^{1} 2\left(r^{2}|u||\nabla u|+r u^{2}\right)(r, \theta) d r \\
& =\int_{0}^{1} 2\left(r|u||\nabla u|+u^{2}\right)(r, \theta) r d r
\end{aligned}
$$

integrando de 0 a $2 \pi$

$$
\begin{aligned}
\int_{0}^{2 \pi}|u(1, \theta)|^{2} d \theta & \leq 2 \int_{0}^{2 \pi}\left(\int_{0}^{1}\left(|u||\nabla u|+u^{2}\right)(r, \theta) r d r\right) d \theta \\
& =2 \int_{\Omega}\left(|u||\nabla u|+u^{2}\right) d x d y
\end{aligned}
$$

Como

$$
\int_{\partial \Omega}|u|^{2} d \theta=\int_{0}^{2 \pi}|u(1, \theta)|^{2} d \theta=\|u\|_{L^{2}(\partial \Omega)}^{2},
$$

y usando la desigualdad de Schwarz tenemos

$$
\begin{aligned}
\|u\|_{L^{2}(\partial \Omega)}^{2} & \leq 2 \int_{\Omega}|u \| \nabla u| d x d y+2 \int_{\Omega} u^{2} d x d y \\
& \leq 2\left(\int_{\Omega}|u|^{2} d x d y\right)^{1 / 2}\left(\int_{\Omega}|\nabla u|^{2} d x d y\right)^{1 / 2}+2 \int_{\Omega} u^{2} d x d y \\
& =2\|u\|_{L^{2}(\Omega)}\|\nabla u\|_{L^{2}(\Omega)}+2\|u\|_{L^{2}(\Omega)}^{2} \\
& =2\|u\|_{L^{2}(\Omega)}\left[\|\nabla u\|_{L^{2}(\Omega)}+\|u\|_{L^{2}(\Omega)}^{2}\right], \text { entonces } \\
\|u\|_{L^{2}(\partial \Omega)}^{2} & \leq 2\|u\|_{L^{2}(\Omega)}\left[\|\nabla u\|_{L^{2}(\Omega)}+\|u\|_{L^{2}(\Omega)}^{2}\right] .
\end{aligned}
$$


Como

$$
\begin{aligned}
\|\nabla u\|_{L^{2}(\Omega)}+\|u\|_{L^{2}(\Omega)} & \leq\left(2\|\nabla u\|_{L^{2}(\Omega)}^{2}+2\|u\|_{L^{2}(\Omega)}^{2}\right)^{1 / 2} \\
& =\left(2 \int_{\Omega}|\nabla u|^{2} d x d y+2 \int_{\Omega}|u|^{2} d x d y\right)^{1 / 2} \\
& =\left(2 \int_{\Omega}\left(|\nabla u|^{2}+|u|^{2}\right) d x d y\right)^{1 / 2} \\
& =2^{1 / 2}\left(\int_{\Omega}\left(\left(\frac{\partial u}{\partial x}\right)^{2}+\left(\frac{\partial u}{\partial y}\right)^{2}+u^{2}\right) d x d y\right)^{1 / 2} \\
& =2^{1 / 2}\|u\|_{H^{1}(\Omega)} .
\end{aligned}
$$

Luego

$$
\|u\|_{L^{2}(\partial \Omega)} \leq 8^{1 / 4}\|u\|_{L^{2}(\Omega)}^{1 / 2}\|u\|_{H^{1}(\Omega)}^{1 / 2}
$$

Hemos probado esta desigualdad para funciones $u$ regulares. Pero veremos que esta desigualdad tiene sentido para todo $u \in H^{1}(\Omega)$ y en forma correspondiente para las funciones de este espacio, la restricción $\left.u\right|_{\partial \Omega}$ tiene sentido como una función de $L^{2}(\partial \Omega)$.

En el ejemplo anterior podemos identificar $L^{2}(\partial \Omega) \operatorname{con} L^{2}([0,2 \pi])$ usando la aplicación $\theta \rightarrow$ $(\cos \theta, \operatorname{sen} \theta)$.

Proposición 2.1 Sea $\Omega$ el disco unitario en $\mathbb{R}^{2}$. Para todo $u \in H^{1}(\Omega)$, la restricción $\left.u\right|_{\partial \Omega}$ puede ser interpretado como una función en $L^{2}(\partial \Omega)$ y satisface la desigualdad (2.7).

\section{Prueba.}

Como $\mathcal{C}^{1}(\bar{\Omega})$ es denso en $H^{1}(\Omega)$, elegimos una sucesión $\left(u_{j}\right)$ en $\mathcal{C}^{1}(\bar{\Omega})$ tal que

$$
\left\|u-u_{j}\right\|_{H^{1}(\Omega)} \leq \frac{1}{j}, \forall j
$$

Por (2.7) y la desigualdad triangular se tiene

$$
\begin{aligned}
\left\|u_{k}-u_{j}\right\|_{L^{2}(\partial \Omega)} & \leq \sqrt[4]{8}\left\|u_{k}-u_{j}\right\|_{L^{2}(\Omega)}^{1 / 2}\left\|u_{k}-u_{j}\right\|_{H^{1}(\Omega)}^{1 / 2} \\
& \leq \sqrt[4]{8}\left\|u_{k}-u_{j}\right\|_{H^{1}(\Omega)} \\
& \leq \sqrt[4]{8}\left[\left\|u_{k}-u\right\|_{H^{1}(\Omega)}+\left\|u-u_{j}\right\|_{H^{1}(\Omega)}\right] \\
& \leq \sqrt[4]{8}\left[\frac{1}{j}+\frac{1}{k}\right] \forall j, \quad \forall k .
\end{aligned}
$$

Luego $\left(u_{j}\right)$ es una sucesión de Cauchy en $L^{2}(\partial \Omega)$. Como este espacio es completo, existe $\nu \in L^{2}(\partial \Omega)$ tal que

$$
\left\|\nu-u_{j}\right\|_{L^{2}(\partial \Omega)} \rightarrow 0 \text { cuando } j \rightarrow \infty \text {. }
$$

Definimos

$$
\left.u\right|_{\partial \Omega}=\nu .
$$

Necesitamos verificar que esta definición no depende de la sucesión particular que elegimos. Así que suponemos que $\left(\nu_{j}\right)$ es otra sucesión en $\mathcal{C}^{1}(\bar{\Omega})$ tal que $\left\|u-\nu_{j}\right\|_{H^{1}(\Omega)} \rightarrow 0$ cuando $j \rightarrow \infty$, por la desigualdad triangular y por (2.7)

$$
\begin{aligned}
\left\|\nu-\nu_{j}\right\|_{L^{2}(\partial \Omega)} & \leq\left\|\nu-u_{j}\right\|_{L^{2}(\partial \Omega)}+\left\|u_{j}-\nu_{j}\right\|_{L^{2}(\partial \Omega)} \\
& \leq\left\|\nu-u_{j}\right\|_{L^{2}(\partial \Omega)}+\sqrt[4]{8}\left\|u_{j}-\nu_{j}\right\|_{H^{1}(\Omega)} \\
& \leq\left\|\nu-u_{j}\right\|_{L^{2}(\partial \Omega)}+\sqrt[4]{8}\left(\left\|u_{j}-u\right\|_{H^{1}(\Omega)}+\left\|u-\nu_{j}\right\|_{H^{1}(\Omega)}\right)
\end{aligned}
$$


La expresión del lado derecho de la desigualdad tiende a 0 cuando $j \rightarrow \infty$. Por lo tanto $\left.u\right|_{\partial \Omega}$ esta bien definido en $L^{2}(\partial \Omega)$. Por otro lado se tiene

$$
\left|\|\nu\|_{L^{2}(\partial \Omega)}-\left\|u_{j}\right\|_{L^{2}(\partial \Omega)}\right| \leq\left\|\nu-u_{j}\right\|_{L^{2}(\partial \Omega)},
$$

y de (2.9) $\|\nu\|_{L^{2}(\partial \Omega)}=\lim _{j \rightarrow \infty}\left\|u_{j}\right\|_{L^{2}(\partial \Omega)}$. También de (2.7) y de (2.8) se tiene

$$
\begin{aligned}
\lim _{j \rightarrow \infty}\left\|u_{j}\right\|_{L^{2}(\partial \Omega)} & \leq \lim _{j \rightarrow \infty} \sqrt[4]{8}\left\|u_{j}\right\|_{L^{2}(\Omega)}^{1 / 2}\left\|u_{j}\right\|_{H^{1}(\Omega)}^{1 / 2} \\
& =\sqrt[4]{8}\|u\|_{L^{2}(\Omega)}^{1 / 2}\|u\|_{H^{1}(\Omega)}^{1 / 2} . \text { Entonces } \\
\|u\|_{L^{2}(\partial \Omega)} & \leq \sqrt[4]{8}\|u\|_{L^{2}(\Omega)}^{1 / 2}\|u\|_{H^{1}(\Omega)}^{1 / 2} .
\end{aligned}
$$

Observación 2.1 Notemos que la proposición (2.1) no define puntualmente $u$ sobre $\partial \Omega$, pero si afirma que $\left.u\right|_{\partial \Omega} \in L^{2}(\partial \Omega)$. Existe la posibilidad que $u$ podría ser infinito en un subconjunto denso de puntos sobre $\partial \Omega$. En lo que sigue describiremos una generalización de la proposición (2.1) a dominios más complejos.

\section{Condición de regularidad en la frontera de un dominio.}

Introduciremos una condición de regularidad sobre la frontera $\partial \Omega$ del dominio $\Omega$.

Definición 3.1. Sea $f \in L^{\infty}(\Omega)$, definiremos la norma de Lipchitz para $f$, denotado por $\|f\|_{\text {Lip }(\Omega)}$, como

$$
\|f\|_{L i p(\Omega)}=\|f\|_{L^{\infty}(\Omega)}+\sup _{x, y \in \Omega, x \neq y} \frac{|f(x)-f(y)|}{|x-y|}
$$

el correspondiente espacio de funciones de Lipschitz es

$$
\operatorname{Lip}(\Omega)=\left\{f \in L^{\infty}(\Omega):\|f\|_{\operatorname{Lip}(\Omega)}<\infty\right\}
$$

Definición 3.2. Sea $\Omega \subset \mathbb{R}^{n}$ un dominio, diremos que $\Omega$ tiene frontera de Lipchitz si existen, una colección de conjuntos abiertos $O_{i}$, un número real $\varepsilon>0$, un número natural $N$ y un número real $M>0$ tal que para todo $x \in \partial \Omega$ la bola de radio $\varepsilon$ con centro $x$ esta contenido en algún $O_{i}$ pero no más de $N$ conjuntos $O_{i}$ que tienen una intersección no vacía con $\Omega$, y para cada uno de estos $O_{i} \cap \Omega=O_{i} \cap \Omega_{i}$, donde $\Omega_{i}$ es un dominio cuya frontera es el gráfico de una función de Lipschitz $\Phi_{i}$ sutisfaciendo $\left\|\Phi_{i}\right\|_{\text {Lip }\left(\mathbb{R}^{n-1}\right)} \leq M$.

El conjunto $\Omega_{i}$ es

$$
\Omega_{i}=\left\{(x, y) \in \mathbb{R}^{n}, x \in \mathbb{R}^{n-1}, y<\Phi_{i}(x)\right\}
$$

Definición 3.3. Diremos que $\partial G \in \mathcal{C}^{0,1}, G \subset \mathbb{R}^{N}$ si la frontera $\partial G$ se puede cubrir con un número finito de superficies $F_{i}, N-1$ dimensionales, las cuales en los sistemas de coordenadas locales adecuadamente elegidos $\left(\xi_{1}, \ldots, \xi_{N}\right)$ tiene la representación

$$
\xi_{N}=g_{i}\left(\xi_{1}, \ldots, \xi_{N-1}\right)
$$

donde $g_{i} \in \mathcal{C}^{0.1}$ para $\left\|\left(\xi_{1}, \ldots, \xi_{N-1}\right)\right\| \leq r_{i}$ con $r_{i}>0$. Se describe esto brevemente diciendo que la frontera $\partial G$ es una variedad $\mathcal{C}^{0,1}$ de dimensión $N-1$ con $G$ localmente a un lado de $\partial G$.

Definición 3.4. Sea $f: G \subset \mathbb{R}^{N} \rightarrow \mathbb{R}$, una función definida sobre una región acotada. $G$ no vacía. Definimos la siguiente norma

$$
\|f\|_{0,1}=\sup _{p \in G}|f(p)|+\sup _{p, q \in G, p \neq q} \frac{|f(p)-f(q)|}{|p-q|},
$$

con el cual podemos definir el siguiente conjunto

$$
\mathcal{C}^{0,1}(\bar{G})=\left\{f \in \mathcal{C}(\bar{G}):\|f\|_{0,1}<\infty\right\} \text {. ver }[2]
$$


Teorema 3.1 Sea $\Omega$ un dominio acotado en $\mathbb{R}^{n}, n \geq 1$ y $\partial \Omega \in \mathcal{C}^{0,1}$ entonces existe un operador lineal continuo

$$
B: H^{1}(\Omega) \rightarrow L^{2}(\partial \Omega)
$$

con la propiedad de que para cada $u \in \mathcal{C}^{1}(\bar{\Omega})$ la función $B u: \partial \Omega \rightarrow \mathbb{R}$ es la función de frontera clásica de $u$, es decir $B u$ es la restricción de $u: \bar{\Omega} \rightarrow \mathbb{R}$ a la frontera $\partial \Omega$.

El caso en el cual $u \in H^{1}(\Omega)$ y $B u \in L^{2}(\partial \Omega)$ se llama función de frontera generalizada en $u$.

Si $u \in H_{0}^{1}(\Omega)$, entonces $B u=0$ en $L^{2}(\partial \Omega)$ es decir $B u(x)=0$ para casi todo $x \in \partial \Omega$.

Observación 3.1 El operador frontera $B$ es importante para la formulación de condiciones de frontera. en el sentido generalizado. Por ejemplo, sea $u \in H^{1}(\Omega)$ y $g \in H^{1}(\Omega)$. La condición de frontera.

$$
u=g \text { en } \partial \Omega
$$

se entiende en el sentido

$$
B u=B g \text { en } L^{2}(\partial \Omega) \text {. ver [5] }
$$

Es decir $B u(x)=B g(x)$ para casi todo $x \in \partial \Omega$. La construcción del operador es como sigue Primero se debe probar la desigualdad

$$
\|u\|_{L^{2}(\partial \Omega)} \leq \text { const. }\|u\|_{H^{1}(\Omega)} \forall u \in \mathcal{C}^{1}(\bar{\Omega})
$$

o equivalentemente

$$
\int_{\partial \Omega} u^{2} d s \leq \text { const. } \int_{\Omega}\left(u^{2}+\sum_{i=1}^{N}\left(D_{i} u\right)^{2}\right) d x
$$

Si $B u$ denota la restricción de $u: \bar{\Omega} \rightarrow \mathbb{R}$ a la frontera $\partial \Omega$ en el caso $u \in \mathcal{C}^{1}(\bar{\Omega})$, entonces $(3.1)$ puede ser escrito como

$$
\|B u\|_{L^{2}(\partial \Omega)} \leq \text { const. }\|u\|_{H^{1}(\Omega)}, \forall u \in \mathcal{C}^{1}(\bar{\Omega})
$$

como $\mathcal{C}^{1}(\bar{\Omega})$ es denso en $H^{1}(\Omega)$ el operador lineal

$$
B: \mathcal{C}^{1}(\bar{\Omega}) \subset H^{1}(\Omega) \rightarrow L^{2}(\partial \Omega)
$$

se puede extender de manera única a u operador lineal continuo

$$
B: H^{1}(\Omega) \rightarrow L^{2}(\partial \Omega), \text { ver }[1]
$$

tal que (3.1) se cumple para todo $u \in H^{1}(\Omega)$. Explícitamente, obtenemos $B u$ de:

Sea $u \in H^{1}(\Omega)$, elegimos una sucesión $\left(u_{n}\right)$ en $\mathcal{C}^{1}(\bar{\Omega})$ tal que $\left\|u_{n}-u\right\|_{H^{1}(\Omega)} \rightarrow 0$, cuando $n \rightarrow \infty$. Entonces $\left\|B u_{n}-B u\right\|_{L^{2}(\partial \Omega)} \rightarrow 0$, cuando $n \rightarrow \infty$ o equivalentemente

$$
\int_{\partial \Omega}\left(u_{n}-B u\right)^{2} d s \rightarrow 0, \text { cuando } n \rightarrow \infty .
$$

\section{Demostración del teorema 3.1 en $\mathbb{R}$.}

Sean $a$ y $b$ números reales, $\bar{\Omega}=[a, b]$ y sea $u \in \mathcal{C}^{\infty}(\bar{\Omega})$. Por el teorema del valor medio

$$
(b-a)^{-1} \int_{a}^{b} u(t) d t=u\left(x_{0}\right) \text { para algún } x_{0} \in[a, b]
$$

entonces $u(x)=\int_{x_{0}}^{x} u^{\prime}(t) d t+(b-a)^{-1} \int_{a}^{b} u(t) d t$

Denotemos $\|u\|_{\mathcal{C}}=\operatorname{máx}_{a \leq x \leq b}|u(x)|$, para todo $u \in \mathcal{G}^{\infty}(\bar{\Omega})$. Por la desigualdad del Hölder se tiene

$$
\begin{aligned}
\|u\|_{\mathcal{C}} & \leq c\left(\int_{a}^{b} u^{\prime 2}(t) d t\right)^{1 / 2}+c\left(\int_{a}^{b} u^{2}(t) d t\right)^{1 / 2} \\
& \leq 2 c\|u\|_{H^{1}(\Omega)}, \text { para alguna constante } c
\end{aligned}
$$


Entonces

$$
u(a)^{2}+u(b)^{2} \leq 2\|u\|_{\mathcal{C}}^{2} \leq 8 c^{2}\|u\|_{H^{1}(\Omega)}^{2} .
$$

Como $\mathcal{C}^{\infty}(\Omega)$ es denso en $H^{1}(\Omega)$ existe una única función continua $j: H^{1}(\Omega) \rightarrow \mathcal{C}(\bar{\Omega})$ con $j(u)=u$, es decir $j(u)(x)=u(x)$ para casi todo $x \in \bar{\Omega}$; los valores de frontera de $u$ son dados por $j(u)(a), j(u)(b)$. Como $\mathcal{C}^{\infty}(\Omega)$ es denso en $H^{1}(\Omega)$, el paso al límite prueba que (3.3) se cumple para todo $u \in H^{1}(\Omega)$; donde después de tomar el límite, $u(a)$ y $u(b)$ se entienden como valores de frontera generalizadas.

\section{Demostración del teorema 3.1 en $\mathbb{R}^{n}$.}

Para $u \in \mathcal{C}^{1}(\bar{\Omega})$, demostraremos que

$$
\int_{\partial \Omega} u^{2} d s \leq c \int_{\Omega}\left[u^{2}+\sum_{j=1}^{N}\left(D_{j} u\right)^{2}\right] d x
$$

Para simplificar la notación consideremos el caso $n=2$. En el caso general se procede análogamente. En una vecindad de un punto $x \in \partial \Omega$ elegimos un sistema de coordenada local $(\xi, \eta)$ donde la frontera tiene la representación local

$$
\eta=\Phi(\xi),-a \leq \xi \leq a
$$

y $\Phi$ es una función de Lipschitz y continua (aplicamos aqui las condiciones de regularidad que se dio en las definiciones (3.1) y (3.2)). Existe $\beta>0$ tal que el conjunto $S$ de todos los puntos $(\xi, \eta)$ donde $-a \leq \xi \leq a, \Phi(\xi)-\beta \leq t \leq \Phi(\xi)$ esta contenido en $\bar{\Omega}$. Sea $u \in \mathcal{C}^{1}(\bar{\Omega})$ entonces

$$
u(\xi, \Phi(\xi))=\int_{t}^{\Phi(\xi)} u_{\eta}(\xi, \eta) d \eta+u(\xi, t)
$$

donde $\Phi(\xi)-\beta \leq t \leq \Phi(\xi)$. Aplicamos la desigualdad $(A+B)^{2} \leq 2 A^{2}+2 B^{2}$ y la desigualdad de Hölder:

$$
u(\xi, \Phi(\xi))^{2} \leq 2 \beta \int_{\Phi(\xi)-\beta}^{\Phi(\xi)} u_{\eta}(\xi, \eta)^{2} d \eta+2 u(\xi, t)^{2} .
$$

Integrando con respecto a $t$ tenemos

$$
\beta u(\xi, \Phi(\xi))^{2} \leq 2 \int_{\Phi(\xi)-\beta}^{\Phi(\xi)}\left[\beta^{2} u_{\eta}(\xi, \eta)^{2}+u(\xi, \eta)^{2}\right] d \eta
$$

Finalmente, integrando sobre el intervalo $[-a, a]$ se tiene

$$
\begin{aligned}
\int_{-a}^{a} \beta u(\xi, \Phi(\xi))^{2} d \xi & \leq 2 \int_{S}\left(\beta^{2} u_{\eta}^{2}+u^{2}\right) d x \\
& \leq c_{1} \int_{S}\left(u_{\xi}^{2}+u_{\eta}^{2}+u^{2}\right) d x .
\end{aligned}
$$

Primero supongamos que $\xi \in \mathcal{C}^{1}$, que $\gamma$ es la curva descrita por $\Phi$, entonces

$$
\int_{\gamma} u^{2} d s=\int_{-a}^{a} u(\xi, \Phi(\xi))^{2} \sqrt{1+\Phi^{\prime}(\xi)^{2}} d \xi
$$

y existe una constante $c_{2}$ tal que $\sqrt{1+\Phi^{\prime}(\xi)^{2}} \leq c_{2}$; luego

$$
\int_{\gamma} u^{2} d s \leq c_{2} \int_{-a}^{a} u(\xi, \Phi(\xi))^{2} d \xi
$$

Si $\Phi$ es una función de Lipschitz continua, entonces $\Phi^{\prime}(\xi)$ existe para casi todo $\xi$. Por otro lado

$$
\operatorname{Lip}(-a, a) \subset W_{\infty}^{1}(-a, a)=\left\{f \in L_{L o c}^{1}(-a, a): \operatorname{máx}_{|a| \leq 1}\left\|D^{\alpha} f\right\|_{L^{\infty}(-a, a)}<\infty\right\}
$$


Luego existe una constante $c_{2}$ tal que

$$
\int_{\gamma} u^{2} d s \leq c_{2} \int_{-a}^{a} u(\xi, \Phi(\xi))^{2} d \xi
$$

De (3.5)

$$
\begin{aligned}
\int_{-a}^{a} u(\xi, \Phi(\xi))^{2} d \xi & \leq \frac{c_{1}}{\beta} \int_{S}\left(u_{\xi}^{2}+u_{\eta}^{2}+u^{2}\right) d x \\
& \leq \frac{c_{1}}{\beta} \int_{\Omega}\left(u_{\xi}^{2}+u_{\eta}^{2}+u^{2}\right) d x
\end{aligned}
$$

De (3.6) y (3.7), para alguna constante $c$ se tiene

$$
\int_{\gamma} u^{2} d s \leq c \int_{\Omega}\left(u_{\xi}^{2}+u_{\eta}^{2}+u^{2}\right) d x=c\|u\|_{H^{1}(\Omega)}^{2} .
$$

Como la frontera $\partial \Omega$ puede ser cubierta por un número finito de vecindades en cada una de las cuales la frontera tiene representación local, sumando las desigualdades correspondientes a (3.8) se obtiene $\int_{\partial \Omega} u^{2} d s \leq c\|u\|_{H^{1}(\Omega)}^{2}$.

\section{Conclusiones}

1. Al estudiar las soluciones de las ecuaciones diferenciales se plantea con frecuencia condiciones a las cuales la solución debe satisfacer en cierta superficie fijada $(n-1)$ - dimensional, por ejemplo en $\partial \Omega$. Es por ello que se necesita generalizar la noción del valor que toma una función definida en c.t.p. de una superficie $n$ - 1- dimensional $S$, esto es la noción de la traza de una función en $S$.

2. Hemos demostrado para el caso $S=\partial \Omega$, que se obtiene para una función $f \in H^{1}(\Omega)$ la correspondiente función $\left.f\right|_{\partial \Omega}$ en $L^{2}(\partial \Omega)$, que le llamamos traza de $f$ en la superficie $(n-1)-$ dimensional $\partial \Omega$ (o función generalizada de frontera).

3. Se generaliza la fórmula de integración por partes, para las funciones $f, g$ en $H^{1}(\Omega)$

$$
\int_{\Omega} f_{x_{i}} g d x=\int_{\partial \Omega} f g \eta_{i} d s-\int_{\Omega} f g_{x_{i}} d x
$$

donde $\eta_{i}=\cos \left(\eta, x_{i}\right)$ es el coseno del ángulo entre la normal $\eta$ exterior a la superficie $\partial \Omega$ y el eje $x_{i}$ mientras que las funciones $f$ y $g$ que se encuentran bajo el signo integral en $\partial \Omega$ son las trazas de estas funciones en $\partial \Omega$. 


\section{REFERENCIAS BIBLIOGRÁFICAS}

[1] Adams,R.A. Sobolev Spaces. Acad. Press, New York, (1975).

[2] Brenner, S.C., Ridgway Scott, L. The Mathematical Theory of Finite Element Methods. Springer - Verlag, New York, (1994).

[3] Medeiros, L.A.,Miranda, M. Espacios de Sobolev. Instituto de Matemática,UFRJ,(1999).

[4] Teman. Roger. Infinite-Dimensional Dynamical Systems in Mechanics and Physics. Springer - Verlag, New York, (1997) .

[5] Zeidler,E. Non Linear Functional Analysis and its applications. Springer - Verlag, New York, (1990). 